\title{
Preferred muscles in cervical dystonia
}

\author{
Wolfgang H. Jost ${ }^{1}$, Bo Biering-Sørensen ${ }^{2}$, Artur Drużdż ${ }^{3}$, Alexandre Kreisler ${ }^{4}$, \\ Sanjay Pandey ${ }^{5}$, Jarosław Sławek ${ }^{6}$, Laurent Tatu ${ }^{7}$ \\ ${ }^{1}$ Parkinson-Klinik Ortenau, Wolfach, Germany \\ ${ }^{2}$ Movement Disorder Clinic, Department of Neurology, Rigshospitalet, Copenhagen, Denmark \\ ${ }^{3}$ Department of Neurology, Municipal Hospital in Poznan, Poland \\ ${ }^{4}$ Service de Neurologie et Pathologie du Mouvement, Chru de Lille, France \\ ${ }^{5}$ Department of Neurology, Govind Ballabh Pant Institute of Postgraduate Medical Education and Research, \\ JLN Marg, New Delhi, India \\ ${ }^{6}$ Department of Neurological-Psychiatric Nursing, Medical University of Gdansk, Department of Neurology, \\ St. Adalbert Hospital, Gdansk, Poland \\ ${ }^{7}$ Department of Neuromuscular Diseases and Department of Anatomy. CHRU Besançon, \\ University of Franche-Comté, Besançon, France
}

\begin{abstract}
The classification of abnormal posture and the assessment of the affected muscles in cervical dystonia (CD) have changed in recent years. To determine the frequency of injected muscles, we studied 306 patients with CD. The mean age was $55.5 \pm 13.1$ years (range 21-90), 67\% were female. Splenius capitis was the most commonly injected muscle (83\%), followed by sternocleidomastoid (79.1\%), and trapezius muscles (58.5\%). The three next most common were the levator scapulae, semispinalis capitis, and obliquus capitis inferior muscles. The study shows that the most commonly injected muscles have remained unchanged over the past few decades, although the concept has changed. However, several new muscles have been added that were previously never, or hardly ever, considered.
\end{abstract}

Key words: cervical dystonia, torticollis, sternocleidomastoid muscle

(Neurol Neurochir Pol 2020; 54 (3): 277-279)

\section{Introduction}

For a long time, the injection scheme in so-called spasmodic torticollis was relatively standardised, with only a few muscles routinely injected. This was because only four basic patterns of $\mathrm{CD}$ were recognised: torticollis, laterocollis, anterocollis and retrocollis. Furthermore, in most countries, approval was only valid for rotary torticollis. Sternocleidomastoid, splenius capitis and trapezius muscles were recommended and injected most frequently [1]. With increasing experience, the injection patterns became more complex and, at least since the introduction of the Col-Cap concept, other muscles such as the obliquus capitis inferior and levator scapulae have also been taken into account $[2,3]$.

In our study, we examined which muscles are injected most frequently in daily clinical practice, regarding the new Col-Cap concept which has distinguished 11 new patterns. These new patterns include the movements of the head (caput) and neck (collis) and/or their combinations [2]. The most common patterns according to this new approach are: torticaput (49\%) and laterocaput (16.7\%). All other subtypes were less than $10 \%$ of the study population [5].

\section{Patients and methods}

Between January and June 2019, we examined prospectively, in seven centres specialized in movement disorders, 306 patients with CD. Patients were included if they had idiopathic CD with pronounced symptoms that interfered with their daily activities, and had been admitted at least three months after their previous BoNT treatment, the effect of which had worn off. The centres were Besançon (France),

Address for correspondence: Wolfgang Jost, Parkinson-Klinik Ortenau, Kreuzbergstr. 12, 77709 Wolfach, Germany, e-mail: W.Jost@parkinson-klinik.de 
Table 1. Frequency of injections (all patients)

\begin{tabular}{|rrrrrrrrrr}
\hline SCM & SM & LS & SsCap & SsCer & SCap & SCer & OCI & Trap & Long \\
\hline $79.1 \%$ & $11.1 \%$ & $48.7 \%$ & $38.2 \%$ & $22.9 \%$ & $83.0 \%$ & $6.9 \%$ & $35.3 \%$ & $58.5 \%$ & $16.7 \%$
\end{tabular}

SCM - Sternocleidomastoideus; SM — Scalene muscles; LS — Levator scapulae; SsCap — Semispinalis capitis; SsCer — Semispinalis cervicis; SCap — Splenius capitis; SCer - Splenius cervicis; OCI — Obliquus capitis inferior; Trap - Trapezius; Long - Longissimus. All others: 20.1\%

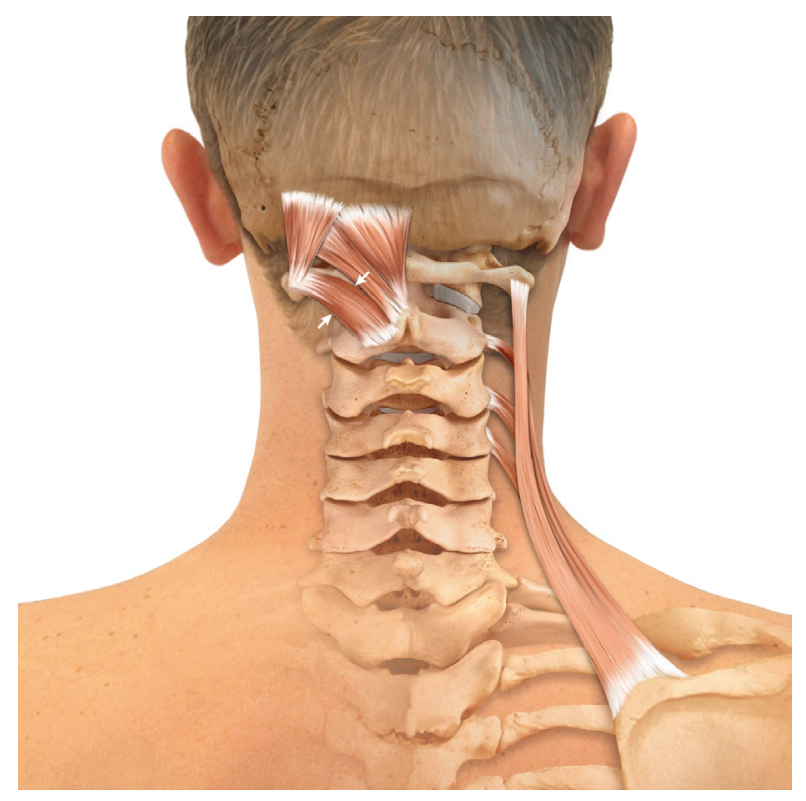

Figure 1. Two muscles that have gained importance through introduction of the Col-Cap concept: obliquus capitis, pictured on the left between the two arrows, and levator scapulae, pictured on the right of the neck

Copenhagen (Denmark), Gdańsk (Poland), Lille (France), New Delhi (India), Poznań (Poland), and Wolfach (Germany). All investigators (WHJ, LT, SP, JS, AD, BBS, AK) are specialists in movement disorders and have long-term experience with BoNT and CD treatment. All injections were performed with the use of ultrasonography guidance. Our study focused on which muscles were most commonly injected.

\section{Results}

306 patients with $\mathrm{CD}$ (mean age $55.5 \pm 13.1$ years, range $21-90,67 \%$ female) were injected and assessed. Splenius capitis was the most common choice in $83 \%$, followed by sternocleidomastoid in $79.1 \%$ and trapezius muscles in $58.5 \%$ (Tab. 1). This was followed by levator scapulae, semispinalis capitis, and obliquus capitis inferior in $38.2 \%, 48.7 \%$ and $35.3 \%$ respectively. The most common primary form was torticaput (49\%), and the second most common was laterocaput (16.7\%) [5].

\section{Discussion}

For a long time, the sternocleidomastoid (SCM), splenius capitis and trapezius muscles were mainly selected to treat cervical dystonia [1]. After the introduction of the Col-Cap concept, it became apparent that the SCM had been overused because it was not involved in torticollis, the supposedly most common form of CD [2-4].

In the past we treated several of the muscles involved, but without distinguishing between caput and collis forms. For example, we treated the sternocleidomastoid muscle because we assumed that it was an important muscle in torticollis. Meanwhile we know that torticaput (but not torticollis) was the most common subtype and that the muscles mentioned, i.e. sternocleidomastoid, splenius capitis and trapezius, were chosen correctly [5]. That explains why the injected muscles may have changed little over the course of the process, although the concept has changed.

It is evident from our study that the muscles mentioned above were injected most frequently. But our study also demonstrates that other muscles previously not recommended should be considered, such as the levator scapulae and obliquus capitis inferior muscles (Fig. 1). The semispinalis cervicis (22.9\%) and the longissimus (16.7\%) are also muscles which have only recently started to be targeted. For some deep muscles (such as the obliquus capitis inferior) or muscles with relatively thin layers (such as the trapezius), the fact that they can only be injected specifically and safely with ultrasound guidance should play a role [6]. In the future, muscles such as the longus colli may also be injected as improved technology combines ultrasonography and electromyography guidance $[7,8]$.

In summary, our study demonstrates that in $\mathrm{CD}$ the same muscles are the ones that are most commonly injected as before, but additional ones, which were rarely taken into account in the past, are now being injected in order to increase the effect of treatment. The Col-Cap concept should be used in everyday clinical practice, and not only in complicated cases and treatment failures.

\section{References:}

1. Jankovic J, Adler CH, Charles D, et al. Primary results from the cervical dystonia patient registry for observation of onabotulinumtoxina efficacy (CD PROBE). J Neurol Sci. 2015; 349(1-2): 84-93, doi: 10.1016/j. jns.2014.12.030, indexed in Pubmed: 25595221.

2. Reichel G. Cervical dystonia: A new phenomenological classification for botulinum toxin therapy. Basal Ganglia. 2011; 1(1): 5-12, doi: 10.1016/j.baga.2011.01.001.

3. Jost WH, Tatu L. Selection of Muscles for Botulinum Toxin Injections in Cervical Dystonia. Mov Disord Clin Pract. 2015; 2(3): 224-226, doi: 10.1002/mdc3.12172, indexed in Pubmed: 30838229. 
4. Tatu L, Jost WH. Anatomy and cervical dystonia. Journal of Neural Transmission. 2016; 124(2): 237-243, doi: 10.1007/s00702-0161621-7.

5. Jost WH, Tatu L, Pandey S, et al. Frequency of different subtypes of cervical dystonia. J Neural Transm. 2020; 127: 45-50.

6. Schramm A, Bäumer T, Fietzek U, et al. Relevance of sonography for botulinum toxin treatment of cervical dystonia: an expert statement. J
Neural Transm (Vienna). 2015; 122(10): 1457-1463, doi: 10.1007/ s00702-014-1356-2, indexed in Pubmed: 25547861.

7. Tyślerowicz M, Jost WH. Injection into the Longus Colli Muscle via the Thyroid Gland. Tremor Other Hyperkinet Mov (N Y). 2019; 9, doi: 10.7916/tohm.v0.718, indexed in Pubmed: 31867133.

8. Tomczykiewicz K. Toksyna botulinowa w leczeniu kręczu karku. Pol Merkur Lekarski. 2016; 41: 107-10. 